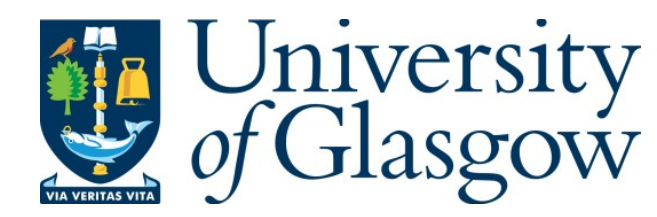

Steinlechner, J., and Martin, I. W. (2016) High index top layer for multimaterial coatings. Physical Review D, 93(10), 102001.

There may be differences between this version and the published version. You are advised to consult the publisher's version if you wish to cite from it.

$\underline{\text { http://eprints.gla.ac.uk/128813/ }}$

Deposited on: 06 October 2016

Enlighten - Research publications by members of the University of Glasgow http://eprints.gla.ac.uk 


\title{
A High Index Top Layer for Multimaterial Coatings
}

\author{
Jessica Steinlechner ${ }^{1}$ and Iain W. Martin ${ }^{1, *}$ \\ ${ }^{1}$ SUPA, School of Physics and Astronomy, University of Glasgow, Glasgow, G12 8QQ, Scotland
}

(Dated: March 4, 2016)

\begin{abstract}
For application in future cryogenically cooled gravitational wave detectors, the thermal noise of low absorbing mirror coatings has to be reduced. The development of low mechanical and optical loss materials is challenging, but thermal noise reduction can be significantly supported by using a multi-material coating design. We analyze the possible improvement of the total (optical and mechanical) loss of a three-material based coating obtained by optimizing the properties of the top layer of the coating stack. A top-layer material with sufficiently high refractive index could have significantly higher optical and mechanical loss than currently used tantala, while still enabling reduction of the total coating loss. Restrictions on possible top-layer material properties are made, and the option of a crystalline top layer is discussed.
\end{abstract}

PACS numbers: 42.25.Bs, 42.79.Wc

\section{INTRODUCTION}

On 14th of September 2015 the first gravitational wave (GW) signal was directly detected by the Advanced LIGO gravitational wave detectors (GWDs) [1], which are large-scale Michelson-type interferometers several kilometers in length. Such detectors have been developed to detect GWs by measuring changes in the relative separation of suspended test-masses via laser interferometry. Highly-reflective (HR) mirror coatings for the test masses are required to have low thermal noise and low optical absorption [2]. To reduce thermal noise, future GW detectors are planned to be operated at cryogenic temperatures. Presently used coating materials, $\mathrm{SiO}_{2}$ and $\mathrm{Ta}_{2} \mathrm{O}_{5}$, show cryogenic mechanical loss peaks and therefore significantly less thermal noise reduction than required when being cooled [3 [5]. To match ET requirements, thermal noise has to be reduced by a factor of $\approx 2$ compared to $\mathrm{SiO}_{2} / \mathrm{Ta}_{2} \mathrm{O}_{5}$ at $20 \mathrm{~K}$.

A promising coating material with low mechanical loss is amorphous silicon (aSi) [6, 7], but the optical absorption at the planned laser wavelength of $1550 \mathrm{~nm}$ is far too high (1000 ppm for a HR coating [8]), while it is only $1.7 \mathrm{ppm}$ [9] for a $\mathrm{SiO}_{2}$ and $\mathrm{Ta}_{2} \mathrm{O}_{5}$ coating. Research on absorption reduction in aSi is ongoing [10]. Additionally, a multimaterial coating design enables the optimization of both optical absorption and mechanical loss [9, 11]. In this design the outermost bilayers are made of two low absorbing materials (e.g. $\mathrm{SiO}_{2}$ and $\mathrm{Ta}_{2} \mathrm{O}_{5}$ ) and reflect most of the incident laser light. Only a small portion of the light is transmitted into the lower layers in which one material (or both) is replaced by a material with lower mechanical loss (e.g. aSi) in which higher absorption can be tolerated because of the reduced power.

A three-material coating made of aSi, $\mathrm{SiO}_{2}$, and $\mathrm{Ta}_{2} \mathrm{O}_{5}$ would reduce the thermal noise at an operation temperature of $20 \mathrm{~K}$ by $25 \%$, while the optical absorption would

\footnotetext{
* iain.martin@glasgow.ac.uk
}
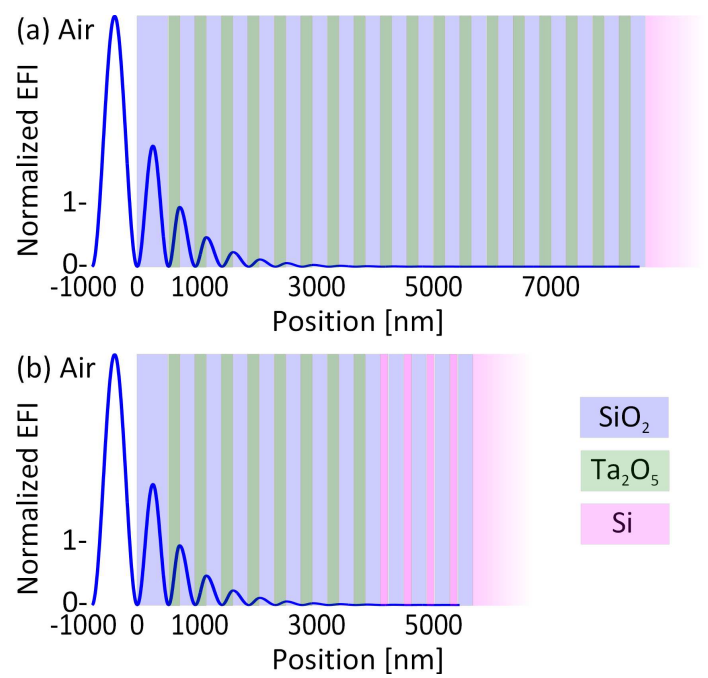

FIG. 1. (a) Schematic of a HR coating stack, which consists of 18 bilayers of $\mathrm{SiO}_{2}$ (blue) and $\mathrm{Ta}_{2} \mathrm{O}_{5}$ (green) on a $\mathrm{Si}$ (pink) substrate. The blue line shows the normalized electric field intensity (EFI) inside the coating. (b) Schematic of a three-material coating identical in reflectivity, in which the outermost 8 bilayers are made of $\mathrm{SiO}_{2}$ and $\mathrm{Ta}_{2} \mathrm{O}_{5}$, while in the lower 4 bilayers $\mathrm{Ta}_{2} \mathrm{O}_{5}$ is replaced by aSi.

increase only by about $3.5 \mathrm{ppm}$ compared to a 'pure' $\mathrm{SiO}_{2} / \mathrm{Ta}_{2} \mathrm{O}_{5}$ coating identical in reflectivity 9 . This is a promising improvement. However, to match the requirements of cryogenic GW detectors, the absorption of the coating has to be further reduced to about $1 \mathrm{ppm}$, and a thermal noise improvement from mechanical loss reduction and/or coating thickness decrease of at least $50 \%$ in total will be necessary.

In this work, the possible thermal noise improvement of the multimaterial design obtained by optimizing the first high-index layer (top layer) of the coating is analyzed. A significant increase of the top-layer refractive index would allow a reduction of the required number of coating layers, while the requirements on mechanical and optical loss of the top-layer material itself would be less stringent. 


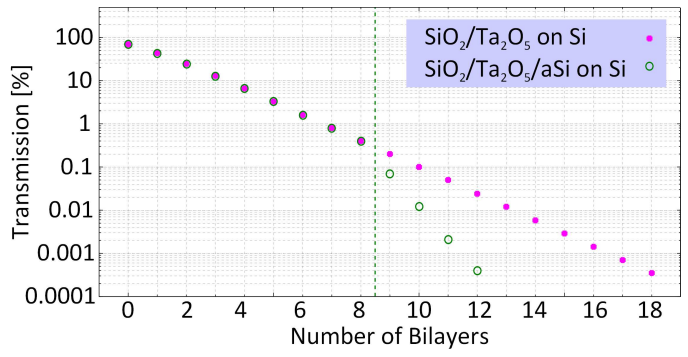

FIG. 2. The transmission decreases after each bilayer: The pink dots show the transmission behind an increasing number of $\mathrm{SiO}_{2} / \mathrm{Ta}_{2} \mathrm{O}_{5}$ bilayers on a $\mathrm{Si}$ substrate (see coating in Fig. 1(a)), the green circles show the transmission after each bilayer for a coating, in which $\mathrm{Ta}_{2} \mathrm{O}_{5}$ is replaced by aSi after the first 8 bilayers. Using aSi requires less bilayers for the two coatings to be identical in reflectivity (see Fig. 1(b)) due to the high refractive index of aSi.

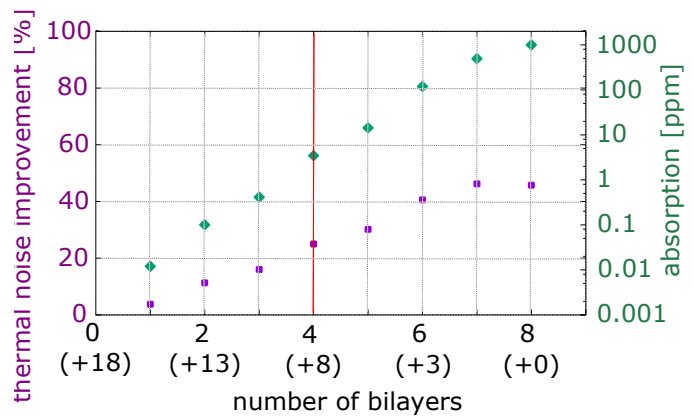

FIG. 3. The relative thermal noise improvement (left y axis) compared to 18 bilayers of $\mathrm{SiO}_{2} / \mathrm{Ta}_{2} \mathrm{O}_{5}$ is shown by the purple squares as a function of the number of used $\mathrm{aSi} / \mathrm{SiO}_{2}$ bilayers at the bottom of the coating stack. In brackets the number of $\mathrm{SiO}_{2} / \mathrm{Ta}_{2} \mathrm{O}_{5}$ required to achieve design reflectivity is given. The green diamonds show the absorption (right y axis) caused by the $\mathrm{aSi} / \mathrm{SiO}_{2}$ bilayers used.

\section{THE MULTI-MATERIAL COATING DESIGN}

HR coatings are based on alternating layers of two materials differing in refractive index $\left(n_{\mathrm{H}}\right.$ for the material with higher refractive index and $n_{\mathrm{L}}$ for the low index material). In the simplest case, to achieve maximum reflectivity each layer has an optical thickness of $n \times t$, where $t$ is the geometrical thickness, of a quarter of a wavelength. For a coating of $\mathrm{N}$ bilayers beginning and ending with a high index layer, a generalized refractive index is given by $n_{\mathrm{G}}=\left(n_{\mathrm{H}}^{2 N+1} / n_{\mathrm{L}}^{2 N}\right) \times n_{\mathrm{S}}$, where $n_{\mathrm{S}}$ is the refractive

TABLE I. Material parameters used for the calculations.

\begin{tabular}{llll}
\hline & $\mathrm{SiO}_{2}$ & $\mathrm{Ta}_{2} \mathrm{O}_{5}$ & $\mathrm{aSi}$ \\
\hline refractive index $n$ & $1.44[12]^{1}$ & $2.05[13]^{2}$ & $3.45[14]^{3}$ \\
mechanical loss ${ }^{*} \phi \times 10^{-4}$ & $7.8[4]$ & $8.6[15]$ & $0.4[6]$ \\
\hline${ }^{1}$ fused silica at $1500 \mathrm{~nm}$ and $30 \mathrm{~K},{ }^{2} \mathrm{Ta}_{2} \mathrm{O}_{5}$ at $1064 \mathrm{~nm}$ and $10 \mathrm{~K}$, \\
${ }^{3} \mathrm{cSi}$ at $1500 \mathrm{~nm}$ and $30 \mathrm{~K},{ }^{*}$ at $20 \mathrm{~K}$ \\
\hline
\end{tabular}

index of the substrate. The reflectivity of this coating is then given by $R=\left(1-n_{\mathrm{G}} / 1+n_{\mathrm{G}}\right)^{2}$ and increases with increasing $\Delta n=\left|n_{\mathrm{H}}-n_{\mathrm{L}}\right|[16]$. While the coatings actually used in GWDs are optimized for thermal noise reduction and consequently differ from quarter layers [17], in this work we concentrate on a standard quarter layer design as the relative thermal noise improvement achieved is very similar for variations in design.

Presently used coatings for GW detectors operating at $1064 \mathrm{~nm}$ are made of almost 40 alternating layers of $\mathrm{SiO}_{2}$ and titania doped $\mathrm{Ta}_{2} \mathrm{O}_{5}$. As the power spectral density of the coating thermal noise is not only proportional to the mechanical loss, but also to the total (geometric) thickness of the coating stack [18], a high refractive index is very beneficial as it simultaneously decreases the number of layers which are required to achieve high reflectivity and the thickness of the layers.

Figure 1(a) shows a schematic of a coating based on alternating quarter wave layers of $\mathrm{SiO}_{2}$ (blue, $t=269 \mathrm{~nm}$ ) and $\mathrm{Ta}_{2} \mathrm{O}_{5}$ (green, $t=189 \mathrm{~nm}$ ). 18 of such bilayers on a Si substrate (pink, $n_{\mathrm{Si}}=n_{\mathrm{S}}=3.45$ ) result in a reflectivity of $\mathrm{R}=99.99965 \%(1-\mathrm{R}=3.5 \mathrm{ppm})$. The first $\mathrm{SiO}_{2}$ layer is half a wavelength in thickness. Such a half wave layer is exploited to optimize thermo-optic noise [19] and does not affect the reflectivity of the coating. In the following discussions it is neglected as thermo-optic noise is not expected to be an issue at cryogenic temperature [20]. In Fig. 1(b) the outermost 8 bilayers are identical to (a), while in the lower bilayers $\mathrm{Ta}_{2} \mathrm{O}_{5}$ was replaced by aSi (pink, $t=112 \mathrm{~nm}$ ). The reflectivity of $\mathrm{R}=99.9996 \%$ (1$\mathrm{R}=4 \mathrm{ppm}$ ) is almost identical to (a), while the coating stack was reduced by 6 bilayers with a total reduction in geometric thickness of $50 \%$. The blue lines show the electric field intensity (EFI) inside the coating.

In Tab. I the refractive indices used for the coating simulations can be found. The refractive index of a coating can vary strongly depending on the deposition method, deposition parameters and treatment after deposition such as heat treatment, and consequently might differ for coatings used in GWDs. As literature for the refractive index of $\mathrm{Ta}_{2} \mathrm{O}_{5}$ at $1550 \mathrm{~nm}$ is rare, a refractive index for cryogenic temperature but $1064 \mathrm{~nm}$ was chosen for our simulations. Small changes of the refractive index of $\mathrm{Ta}_{2} \mathrm{O}_{5}$ do not change the relative improvement of a multimaterial coating compared to a standard $\mathrm{SiO}_{2} / \mathrm{Ta}_{2} \mathrm{O}_{5}$ coating significantly. For aSi, variations in refractive index are bigger and numbers of $\mathrm{n}_{\mathrm{aSi}}>4$ can be found in literature. $\mathrm{n}_{\mathrm{cSi}}=3.45$ (at $1550 \mathrm{~nm}$ at $30 \mathrm{~K}$ ) was chosen as a lower limit providing the smallest possible relative improvement of the coating design presented here compared to a standard $\mathrm{SiO}_{2} / \mathrm{Ta}_{2} \mathrm{O}_{5}$ coating.

In the simplest case, a multimaterial coating is equivalent to two coating stacks on top of each other. In Fig. 2 the transmitted power after each bilayer is shown for the two coatings (1)(a) and (b)); the pink dots represent the ordinary $\mathrm{SiO}_{2} / \mathrm{Ta}_{2} \mathrm{O}_{5}$ coating, while the green circles show the reflectivity for the three-material coating, in which the $\mathrm{Ta}_{2} \mathrm{O}_{5}$ is replaced by aSi below the 8 th bi- 
layer. This change in material is highlighted by the green dashed, vertical line. The upper stack reflects most of the power to allow higher absorption in the lower stack. In our example the power is reduced to $0.4 \%$ allowing the absorption of the lower stack to be higher by a factor of 250 compared to the upper stack for the two coating parts to equally contribute to the total absorption.

The low mechanical loss of aSi, the reduced number of bilayers and the reduced geometric thickness of the aSi layers compared to $\mathrm{Ta}_{2} \mathrm{O}_{5}$ result in about $25 \%$ thermal noise reduction at $20 \mathrm{~K}$ compared to $\mathrm{SiO}_{2} / \mathrm{Ta}_{2} \mathrm{O}_{5}$. The absorption is kept as low as $4 \mathrm{ppm}$ (plus absorption of the $\mathrm{SiO}_{2} / \mathrm{Ta}_{2} \mathrm{O}_{5}$ stack) despite the high aSi absorption. However, to match future GW detector requirements, both parameters have to be further improved. For unchanged material properties, to reduce the optical absorption, the boundary between the upper and the lower stack in Fig. 2 would need to move to the right, while to improve thermal noise, it would need to move to the left.

Figure 3 shows the relative thermal noise improvement of the three material coating in \% (purple squares, left $\mathrm{y}$ axis) compared to 18 bilayers of $\mathrm{SiO}_{2} / \mathrm{Ta}_{2} \mathrm{O}_{5}$ as a function of the number of used aSi $/ \mathrm{SiO}_{2}$ bilayers at the bottom of the coating stack. The number of $\mathrm{SiO}_{2} / \mathrm{Ta}_{2} \mathrm{O}_{5}$ bilayers which is required to keep the reflectivity of the coating constant for the different numbers of $\mathrm{aSi} / \mathrm{SiO}_{2}$ bilayers used is shown in brackets. Only integer numbers of bilayers made of quarter waves were used. The green diamonds show the absorption (right y axis) caused by the $\mathrm{aSi} / \mathrm{SiO}_{2}$ bilayers in the coating. The red line indicates the design discussed in this paper. As shown in Fig. 3. an absorption of $1 \mathrm{ppm}$ can be achieved by a design using $3 \mathrm{aSi} / \mathrm{SiO}_{2}$ bilayers, giving a thermal noise improvement of $18 \%$. Obtaining further thermal noise improvement, without increasing the absorption of the coating, would require either the optical absorption of the aSi layers to be reduced, allowing the boundary to be shifted to the left, the mechanical loss of the $\mathrm{Ta}_{2} \mathrm{O}_{5}$ and/or $\mathrm{SiO}_{2}$ layers to be reduced, or, as discussed in this paper, the effective reduction of the aSi absorption via the use of a high-index top-layer to reduce the light intensity in the stack.

\section{AN ALTERNATIVE TOP LAYER MATERIAL}

Optimizing the first high index layer on top of the stack (top layer), reduces the fraction of the incident light which is transmitted into the lower layers. Figure 4 shows the laser power which is transmitted by the upper stack with increasing refractive index of the top layer. The transmitted power is normalized to the power which would be transmitted for a $\mathrm{Ta}_{2} \mathrm{O}_{5}$ top layer. The blue area indicates a transmission reduction resulting in an absorption reduction in the lower stack. At the border from blue to green $(n=2.92)$ this reduction would allow one $\mathrm{SiO}_{2} / \mathrm{Ta}_{2} \mathrm{O}_{5}$ bilayer to be removed without increasing the absorption of the coating beyond the initial multi-

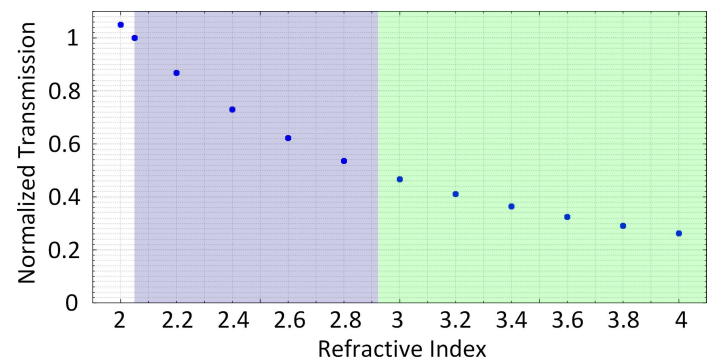

FIG. 4. Transmission of the upper coating stack for a varied refractive index of the first high index layer (normalized to the transmission for the refractive index of $\left.\mathrm{Ta}_{2} \mathrm{O}_{5}\right)$ : With increasing refractive index, the transmission decreases and reduces the absorption in the lower stack. For a top layer refractive index of 2.92 this decrease is equivalent to one additional $\mathrm{SiO}_{2} / \mathrm{Ta}_{2} \mathrm{O}_{5}$ bilayer. For a top layer refractive index of 4.05 it is equivalent to two additional bilayers.

material design, for $n=4.05$ (right end of the graph) it would allow two $\mathrm{SiO}_{2} / \mathrm{Ta}_{2} \mathrm{O}_{5}$ bilayers to be removed.

The changing refractive index also changes the requirements on mechanical loss and optical absorption on the top layer material. The following two sections examine the boundaries for these parameters.

\section{A. Mechanical Loss Requirements on the Top Layer Material}

Since coating thermal noise power spectral density is proportional to $t$ and $\phi$, there will be no change in the thermal noise of the top-layer as long as the product is kept constant. Thus the reduction in geometric thickness of the top layer due to the higher refractive index directly allows an increase of the mechanical loss by $n / n_{\mathrm{Ta}_{2} \mathrm{O}_{5}} \times \phi_{\mathrm{Ta}_{2} \mathrm{O}_{5}}$, while still fully exploiting the loss decrease from reducing the stack by one (two) bilayer(s). As the contribution of one single layer to the total mechanical loss of the coating is small, the restrictions on the top-layer mechanical loss are lower than for the other materials in the coating.

Figure 5 shows the thermal noise percentage improvement compared to an 18 bilayer $\mathrm{SiO}_{2} / \mathrm{Ta}_{2} \mathrm{O}_{5}$ coating (optimized for $1550 \mathrm{~nm}$, on a Si substrate at $20 \mathrm{~K}$ at $100 \mathrm{~Hz}$ ), as a function of the mechanical loss of the top layer. The pink dot indicates the thermal noise improvement which is possible for the multimaterial coating shown in Fig. 1(b). For a top layer with a refractive index of $n=2.92$ the reflectivity of the coating increases so that one $\mathrm{SiO}_{2} / \mathrm{Ta}_{2} \mathrm{O}_{5}$ bilayer can be removed to keep the laser power in the lower stack identical to the initial multimaterial design. The blue line shows the resulting thermal noise improvement (due to the removed bilayer) as a function of the mechanical loss of the top layer. For a top layer with a refractive index of $n=4.05$ a second $\mathrm{SiO}_{2} / \mathrm{Ta}_{2} \mathrm{O}_{5}$ bilayer can be removed. The resulting thermal noise improvement as a function of the mechan- 


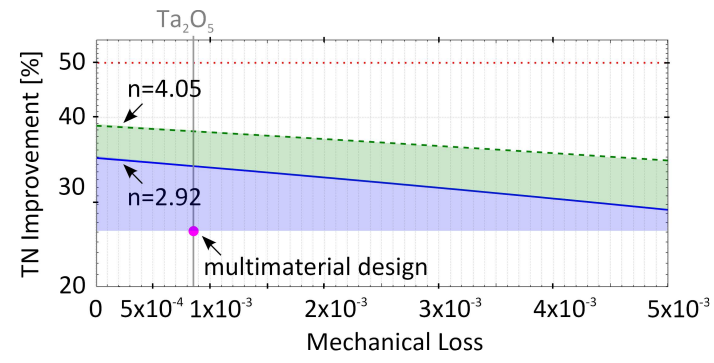

FIG. 5. Thermal noise (TN) improvement normalized to 18 bilayers of $\mathrm{SiO}_{2} / \mathrm{Ta}_{2} \mathrm{O}_{5}$ on a $\mathrm{Si}$ substrate, for the initial multimaterial design (○) 9] (see Fig. 1), for a top layer with a refractive index of $n=2.92$ and an upper stack which was reduced by one $\mathrm{SiO}_{2} / \mathrm{Ta}_{2} \mathrm{O}_{5}$ bilayer (-), and for a top layer with a refractive index of $n=4.05$ and an upper stack which was reduced by two $\mathrm{SiO}_{2} / \mathrm{Ta}_{2} \mathrm{O}_{5}$ bilayers (-

- ), both as a function of the mechanical loss of the top layer. Blue area: TN improvement for refractive indices of $\mathrm{Ta}_{2} \mathrm{O}_{5}<n<2.92$. Green area: TN improvement for refractive indices of $2.92<n<4.05$ and an upper stack which was reduced by one $\mathrm{SiO}_{2} / \mathrm{Ta}_{2} \mathrm{O}_{5}$ bilayer. In addition, the mechanical loss of $\mathrm{Ta}_{2} \mathrm{O}_{5}(\mid)$ and the goal of $50 \% \mathrm{TN}$ reduction $(\cdots)$ are marked.

ical loss of the top layer is shown by the green, dashed line. The blue area indicates the possible thermal noise improvement for $n_{\mathrm{Ta}_{2} \mathrm{O}_{5}}<n<2.92$. In this case no $\mathrm{SiO}_{2} / \mathrm{Ta}_{2} \mathrm{O}_{5}$ can be removed, but the bilayer thickness can be adjusted (i.e. reduced) based on the required reflectivity resulting in a thermal noise improvement. The green area shows the possible thermal noise improvement for $2.92<n<4.05$ and an upper stack which was reduced by one $\mathrm{SiO}_{2} / \mathrm{Ta}_{2} \mathrm{O}_{5}$ bilayer. In this example, the maximum thermal noise improvement for a top layer with the mechanical loss of $\mathrm{Ta}_{2} \mathrm{O}_{5}$ is $25 \%$ for the initial multimaterial coating design, $33 \%$ for a top layer with $n=2.92$, and $38 \%$ for $n=4.05$. The goal of a thermal noise reduction of $50 \%$ is shown by the red, dotted line.

\section{B. Absorption Requirements on the Top Layer Material}

Based on the reduced thickness of the top layer arising from the high refractive index, the absorption coefficient is allowed to increase by a factor of $n / n_{\mathrm{Ta}_{2} \mathrm{O}_{5}}$ for no change in the absorption of the layer. In addition, the EFI in the top layer and in the bilayers below, decreases with increasing refractive index of the top layer, which also allows a higher absorption coefficient. Figure 6)(a) shows the EFI in the upper stack for a fixed coating design identical to Fig. 1(b), in which only the top layer (= first high index layer) refractive index of $n=2.05$ (pink) is changed to $n=2.92$ (blue), and to $n=4.05$ (green).

Figure 6(b) shows the maximum possible absorption coefficient of the top-layer material (normalized to the absorption coefficient of $\mathrm{Ta}_{2} \mathrm{O}_{5}$ on the right $\mathrm{y}$ axis) for which the absorption of the upper stack does not exceed
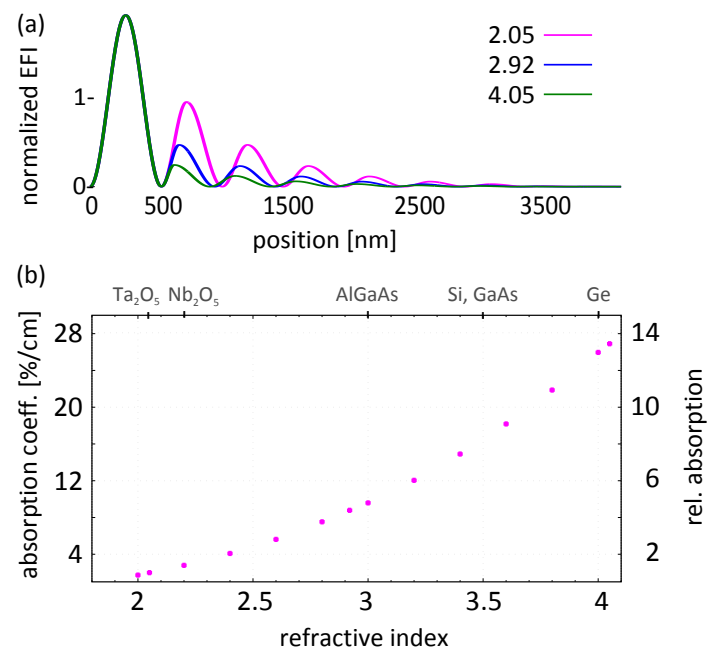

FIG. 6. (a) EFI in the upper stack for a top layer with $n=2.05$ (pink), $n=2.92$ (blue), and $n=4.05$ (green). (b) Maximum possible absorption coefficient for the top layer material for which the total absorption of the upper stack does not exceed the absorption of an upper stack with a $\mathrm{Ta}_{2} \mathrm{O}_{5}$ top layer, as a function of the top-layer refractive index (left yaxis: absolute absorption coefficient, right y-axis: absorption normalized to $\mathrm{Ta}_{2} \mathrm{O}_{5}$ absorption). Above the graph materials with different refractive indices are indicated. Note that these examples for materials refer only to the refractive indices, not to the absorption.

the absorption of a coating with a $\mathrm{Ta}_{2} \mathrm{O}_{5}$ top layer. Examples of materials with different refractive indices are shown along the top of the graph. Note that these examples for materials refer only to the refractive indices, not to the absorption. For these considerations a constant transmission of the upper stack of $0.4 \%$ of the incident light was assumed so that the absorption in the lower stack is kept constant.

While the thermal noise calculations used only toplayer refractive indices which allow the removal of interger numbers of bilayers, the use of refractive indices between these values results in an additional increase of the reflectivity of the upper stack and reduces the transmission into (and therefore the absorption inside) the lower stack. For example, an $n=3.5$ material would reduce the absorption in the lower stack by about $30 \%$.

\section{DISCUSSION}

We have shown that a top layer with high refractive index would significantly improve a coating by reducing the number of bilayers required to achieve high reflectivity (or alternatively to reduce the power transmitted into the lower stack and thereby reducing the absorption).

The intention of Figs. 5 and 6(b) is to give an overview of the suitability of potential materials with apparently poor mechanical and optical properties (compared to $\mathrm{SiO}_{2}$ and $\left.\mathrm{Ta}_{2} \mathrm{O}_{5}\right)$ as a top-layer material, and the sug- 
TABLE II. Thermal noise and optical absorption for different coating designs: Coating A is a commonly used $\mathrm{SiO}_{2} / \mathrm{Ta}_{2} \mathrm{O}_{5}$ coating, coating $\mathrm{B}$ is an $\mathrm{aSi} / \mathrm{SiO}_{2} / \mathrm{Ta}_{2} \mathrm{O}_{5}$ multimaterial coating. The total coating absorption was calculated based on 1000 ppm [8] for a highly-reflective aSi/ $\mathrm{SiO}_{2}$ coating. Coatings $\mathrm{C}$ and $\mathrm{D}$ show the thermal noise improvement and absorption for a cSi top layer and reduction by one (C) and two (D) $\mathrm{SiO}_{2} / \mathrm{Ta}_{2} \mathrm{O}_{5}$ bilayers.

\begin{tabular}{|c|c|c|c|c|}
\hline & $\mathrm{A}$ & $\mathrm{B}$ & $\mathrm{C}$ & $\mathrm{D}$ \\
\hline & $18 \times \mathrm{SiO}_{2} / \mathrm{Ta}_{2} \mathrm{O}_{5}$ & $8 \times \mathrm{SiO}_{2} / \mathrm{Ta}_{2} \mathrm{O}_{5}$ & $1 \times \mathrm{cSi} / \mathrm{SiO}_{2}$ & $1 \times \mathrm{cSi} / \mathrm{SiO}_{2}$ \\
\hline & & $4 \times \mathrm{aSi} / \mathrm{SiO}_{2}$ & $6 \times \mathrm{SiO}_{2} / \mathrm{Ta}_{2} \mathrm{O}_{5}$ & $5 \times \mathrm{SiO}_{2} / \mathrm{Ta}_{2} \mathrm{O}_{5}$ \\
\hline & & & $4 \times \mathrm{aSi} / \mathrm{SiO}_{2}$ & $4 \times \mathrm{aSi} / \mathrm{SiO}_{2}$ \\
\hline Thermal Noise at $100 \mathrm{~Hz} \times 10^{-21}$ & 2.11 & $1.54(-27 \%)$ & $1.42(-32 \%)$ & $1.32(-37 \%)$ \\
\hline Optical Absorption at $1550 \mathrm{~nm}$ & $1.7 \mathrm{ppm}[9]$ & $5.7 \mathrm{ppm}$ & $3.2 \mathrm{ppm}$ & $5.9 \mathrm{ppm}$ \\
\hline
\end{tabular}

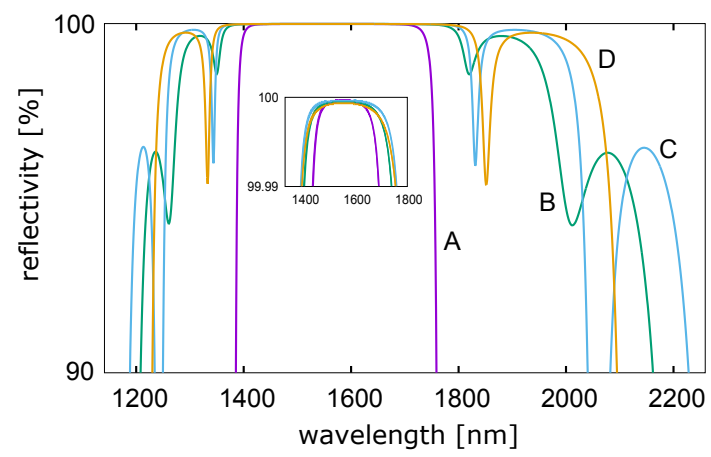

FIG. 7. Reflectivity of the coatings A - D listed in Tab. III Because of the high index silicon component in the coating, the spectral response around the design wavelength of $1550 \mathrm{~nm}$ is flat over a wider wavelength range for coating $\mathrm{B}-\mathrm{D}$ than for the original pure $\mathrm{SiO}_{2} / \mathrm{Ta}_{2} \mathrm{O}_{5}$ coating (A).

gestion of reconsidering materials previously presumed to be unsuitable for application in coatings.

For the material restrictions calculations, we initially considered amorphous materials which often show high optical and mechanical loss. Crystalline materials for coating applications offer an entire new spectrum for material parameter optimization. Crystalline coatings show promising material properties for application in GW detectors. Coatings made of alternating layers of AlGaAs (aluminium gallium arsenide) and GaAs (gallium arsenide) have low mechanical loss 24] and low optical absorption 22]. For fabrication of crystalline coatings, the coating and base substrate materials are required to have matching lattice structures. AlGaAs coatings are grown on GaAs wafers to be later transferred and bonded to substrates of appropriate materials (e.g. fused silica or $\mathrm{Si}$ ), like the coating which was investigated in [22]. The maximum possible diameter of AlGaAs coatings is currently limited to $200 \mathrm{~mm}$ by the size of available GaAs wavers. This is significantly smaller than the design testmass diameter of $\approx 40 \mathrm{~cm}$ generally used for future $\mathrm{GW}$ detectors [2].

Using a single, crystalline top layer would enable exploitation of the advantage of a high refractive index and low optical and mechanical loss, while avoiding the issue of a limited variety of materials with a certain lattice structure encountered with pure crystalline multilayer coatings. An interesting option may be to coat such a crystalline single layer with amorphous layers forming a highly-reflective crystalline and amorphous (c-a) mixed coating. Bonding of the amorphous (back) side of the coating to a substrate would minimize any possible absorption of the 'bonding layer' as the laser power at the bottom of the highly reflective coating is low. It was previously shown that the absorption of a direct bond which is used to apply a coating a fused silica substrate is in the order of magnitude of only a few ppm 24, 25].

Crystalline silicon ( $\mathrm{cSi}$ ) is a potential candidate material due to its low mechanical loss [23, 26], low optical absorption [27, 28], and various industrial applications providing large scale substrates. The mechanical loss of cSi at $20 \mathrm{~K}$ can be as low as $3 \times 10^{-7}$. The optical absorption for a single quarter-wave layer is negligible even for highly doped material for semiconductor applications. A low resistivity (i.e. high doping) of $0.1 \Omega \mathrm{cm}$ would induce an absorption of less than $0.1 \mathrm{ppm}$ per $100 \mathrm{~nm}$ layer [28].

Table II shows the thermal noise and the optical absorption for different coating designs. Coating $\mathrm{A}$ is a commonly used $\mathrm{SiO}_{2} / \mathrm{Ta}_{2} \mathrm{O}_{5}$ coating. Coating $\mathrm{B}$ is the aSi $/ \mathrm{SiO}_{2} / \mathrm{Ta}_{2} \mathrm{O}_{5}$ coating design proposed in [9] based on an optical absorption of $1000 \mathrm{ppm}$ in a highly reflective $\mathrm{aSi} / \mathrm{SiO}_{2}$ coating. In coating design $\mathrm{C}$, the top layer is made of $\mathrm{cSi}$ instead of $\mathrm{Ta}_{2} \mathrm{O}_{5}$ and one $\mathrm{SiO}_{2} / \mathrm{Ta}_{2} \mathrm{O}_{5}$ bilayer is removed. The thermal noise of the coating stack is reduced by $32 \%$ compared to $\mathrm{A}$, while the absorption of $3.2 \mathrm{ppm}$ is significantly lower than for the original multimaterial coating $\mathrm{B}$ (5.7 ppm). In coating design D, two $\mathrm{SiO}_{2} / \mathrm{Ta}_{2} \mathrm{O}_{5}$ bilayers are removed, which reduces the thermal noise of the coating stack by $37 \%$ in total. The absorption of $5.9 \mathrm{ppm}$ is almost identical with the absorption of B. The spectral response (see Fig. 7) of the coatings $\mathrm{B}-\mathrm{D}$ is flat over a wider wavelength range than for the original pure $\mathrm{SiO}_{2} / \mathrm{Ta}_{2} \mathrm{O}_{5}$ coating (A) due to the high refractive index of the aSi component. Therefore this design will ensure high reflectivity over the required bandwidth ensuring the required optical performance can be maintained in the presence of thermal drifts, deposition tolerances and inhomegeneities.

The silicon on insulator (SOI) growth technique might serve as a base for the fabrication of a large scale cSi top layer. For SOI, single cSi layers $100 \mathrm{~nm}$ (and less) 
in thickness are produced separated from the wafer by an oxide layer (e.g. $\mathrm{SiO}_{2}$ ). The oxide layer could serve as a buffer layer to etch off the $\mathrm{Si}$ wafer after having coated and bonded it to a substrate, leaving the oxide layer and the thin cSi top layer in place. The oxide layer either could be etched with a compound, which leaves the Si top layer unaffected or could serve as half wave cap. Wafers of up to $300 \mathrm{~mm}$ in diameter are available and current research concentrates on expanding SOI to a diameter of $450 \mathrm{~mm}$ 29].

We conclude that a high-index top layer, in particular a crystalline layer, would be beneficial for thermal noise reduction in low absorbing, highly-reflective coatings. Further absorption reduction in aSi can significantly improve the absorption performance. To investigate the practicability of a large scale crystalline and amorphous mixed coating, detailed R\&D will be required.

\section{ACKNOWLEDGEMENTS}

We are grateful for financial support from STFC (ST/L000946/1) and the University of Glasgow. I. W. M is supported by a Royal Society Research Fellowship. We are grateful to the International Max Planck Partnership for Measurement and Observation at the Quantum Limit for support, and we thank our colleagues in the LSC and VIRGO collaborations and within SUPA for their interest in this work. This paper has LIGO Document number LIGO-P1500256.
[1] B. P. Abbott et al., Observation of Gravitational Waves from a Binary Black Hole Merger, Phys. Rev. Lett. 116, 061102 (2016)

[2] M. Abernathy et al., Einstein gravitational wave Telescope (ET) conceptual design study, ET-0106C-10, https://tds.ego-gw.it/ql/?c=7954 (2011)

[3] M. Granata et al., Cryogenic measurements of mechanical loss of high-reflectivity coating and estimation of thermal noise, Optics Letters 38, 5268 (2013)

[4] I. W. Martin et al. Low temperature mechanical dissipation of an ion-beam sputtered silica film, Class. Quantum Grav. 31, 035019 (2014)

[5] I. W. Martin et al., Comparison of the temperature dependence of the mechanical dissipation in thin films of $\mathrm{Ta}_{2} \mathrm{O}_{5}$ and $\mathrm{Ta}_{2} \mathrm{O}_{5}$ doped with $\mathrm{TiO}_{2}$, Class. Quantum Grav. 26, 155012 (2009)

[6] P. G. Murray, I. W. Martin, K. Craig, J. Hough, R. Robie, S. Rowan, M. R. Abernathy, T. Pershing, and S. Penn Ion-beam sputtered amorphous silicon films for cryogenic precision measurement systems Phys. Rev. D 92062001 (2015)

[7] X. Liu, C. L. Spiel, R. D. Merithew, R. O. Pohl, B. P. Nelson, Q. Wang, R. S. Crandall, Internal friction of amorphous and nanocrystalline silicon at low temperatures, Materials Science and Engineering A 442 307-313, (2006)

[8] J. Steinlechner, A. Khalaidovski, R. Schnabel, Optical absorption measurement at $1550 \mathrm{~nm}$ on a highlyreflective $\mathrm{Si} / \mathrm{SiO}_{2}$ coating stack, Class. Quantum Grav. 31, 105005 (2014)

[9] J. Steinlechner, I. W. Martin, C. Krueger, J. Hough, S. Rowan, R. Schnabel, Thermal Noise Reduction and Absorption Optimisation via Multi-Material Coatings, Phys. Rev. D 91042001 (2015)

[10] J. Steinlechner, I. W. Martin, R. Bassiri, A. Bell, M. M. Fejer, J. Hough, A. Markosyan, R. K. Route, S. Rowan Optical Absorption of Ion-Beam Sputtered aSi Coatings In preparation

[11] W. Yam, S. Gras, M. Evans, Multimaterial coatings with reduced thermal noise Phys. Rev. D 91042002 (2015)

[12] D. B. Leviton, B. J. Frey Temperature-dependent absolute refractive index measurements of synthetic fused silica Proc. SPIE 6273 Optomechanical Technologies for Astronomy 62732K (2006)

[13] J. Franc, N. Morgado, R. Flaminio, R. Nawrodt, I. Martin, L. Cunningham, A. Cumming, S. Rowan, J. Hough Mirror thermal noise in laser interferometer gravitational wave detectors operating at room and cryogenic temperature arXiv:0912.0107 [gr-qc] (2009)

[14] B. J. Frey, D. B. Leviton, T. J. Madison Temperature dependent refractive index of silicon and germanium arXiv:physics/0606168v1 (2006)

[15] Iain W. Martin et al., Effect of heat treatment on mechanical dissipation in $\mathrm{Ta}_{2} \mathrm{O}_{5}$ coatings, Class. Quantum Grav. 27, 225020 (2010)

[16] H. E. Bennett, D. K. Burke, Simple expressions for predicting the effect of volume and interface absorption and of scattering in high-reflectance or antireflectance multilayer coatings, J. Opt. Soc. Am., Vol. 70, No. 3, (1980)

[17] A. K. Villar, E.D. Black, R. DeSalvo, K. G. Libbrecht, C. Michel, N. Morgado, L. Pinard, I. M. Pinto, V. Pierro, V. Galdi, M. Principe, I. Taurasi, Measurement of thermal noise in multilayer coatings with optimized layer thickness, Phys. Rev. D 81, 122001 (2010)

[18] G. M. Harry et al., Thermal noise in interferometric gravitational wave detectors due to dielectric optical coatings, Class. Quantum Grav. 19, 897-917 (2002)

[19] M. Evans, S. Ballmer, M. Fejer, P. Fritschel, G. Harry, and G. Ogin, Thermo-optic noise in coated mirrors for high-precision optical measurements, Phys. Rev. D 78, 102003 (2008)

[20] K. Yamamoto, Thermo-optic noise at cryogenic temperature, http://gwdoc.icrr.utokyo.ac.jp/DocDB/0018/T1001852/001

[21] G. D. Cole, W. Zhang, M. J. Martin, J. Ye, and M. Aspelmeyer, Tenfold reduction of Brownian noise in highreflectivity optical coatings, Nature Photonics 7 644-650 (2013)

[22] J. Steinlechner, I. W. Martin, A. Bell, G. Cole, J. Hough, S. Penn, S. Rowan, S. Steinlechner, Mapping the optical absorption of a substrate-transferred crystalline AlGaAs coating at $1.5 \mu \mathrm{m}$, Class. Quantum Grav. 32105008 (2015) 
[23] D. F. McGuigan, C. C. Lam, R. Q. Gram, A. W. Hoffman, D. H. Douglass, H. W. Gutche, Measurements of the mechanical Q of single-crystal silicon at low temperatures, Journal of Low Temperature Physics 30 624-629 (1978)

[24] G. D. Cole, W. Zhang, M. J. Martin, J.Ye, M. Aspelmeyer, Tenfold reduction of Brownian noise in highreflectivity optical coatings, Nature Photonics 7 644-650 (2013)

[25] J. Steinlechner, I. W. Martin, Angus Bell, Garrett Cole, Jim Hough, Steven Penn, Sheila Rowan, Sebastian Steinlechner, Mapping the Optical Absorption of a SubstrateTransferred Crystalline AlGaAs Coating at $1.5 \mu \mathrm{m}$, Classical and Quantum Gravity 32105008 (2015)
[26] R. Nawrodt et al., High mechanical Q-factor measurements on silicon bulk samples, Journal of Physics: Conference Series 122012008 (2008)

[27] M. J. Keevers, M. A. Green, Absorption edge of silicon from solar cell spectral response measurements, Appl. Phys. Lett. 66 174-176 (1995)

[28] J. Degallaix, R. Flaminio, D. Forest, M. Granata, C. Michel, L. Pinard, T. Bertrand, G. Cagnoli, Bulk optical absorption of high resistivity silicon at $1550 \mathrm{~nm}$, Optics Letter 3812 2047-2049 (2013)

[29] Development of $450 \mathrm{~mm}$ SOI substrates, related technologies and equipments, Presentation, SEMICON Europa2013, 2013 http://www.semi.org 